\title{
Baryon number violation involving higher generations
}

\author{
Wei-Shu Hou, ${ }^{1,2}$ Makiko Nagashima, ${ }^{1}$ and Andrea Soddu ${ }^{1,3}$ \\ ${ }^{1}$ Department of Physics, National Taiwan University, Taipei, Taiwan 106, Republic of China \\ ${ }^{2}$ Stanford Linear Accelerator Center, Stanford, California 94309, USA \\ ${ }^{3}$ Department of Particle Physics, Weizmann Institute of Science, Rehovot 76100, Israel
}

(Received 1 September 2005; published 3 November 2005)

\begin{abstract}
Proton stability seems to constrain rather strongly any baryon number violating process. We investigate the possibility of baryon number violating processes involving right-handed dynamics or higher generation quarks. Our results strongly suggest that there will be no possibility to observe baryon number violation in $\tau$ or higher generation quark decays, at any future machine.
\end{abstract}

\section{INTRODUCTION}

Proton decay was suggested 30 years ago as a means to probe physics at a very high energy scale, such as [1] grand unified theories. Baryon number violation is also a key ingredient [2] to understand the baryon asymmetry of our Universe. Experimental search for proton decay has yielded [3] only limits so far, albeit very stringent ones, and improvement requires huge underground facilities.

More recently, in an attempt to solve the hierarchy problem [4], a new scenario has been proposed, which assumes the existence of large compactified extra dimensions. It has been observed that, having no test of gravity below the millimeter scale, the radius of compactification can be much larger than $M_{\text {Planck }}^{-1}$, provided the extra dimensions are accessible only to gravitational interactions. Standard model fields are instead localized on a threedimensional wall whose inverse thickness $L^{-1}$ can be of order TeV. Denoting by $V_{n}$ the volume of the extra space, with $n$ the number of compact extra dimensions, and by $M$ the fundamental scale of gravity, the observed Planck mass is given by $M_{\text {Planck }}^{2}=M^{n+2} V_{n}$. By asking that $n \geq 2$, the fundamental scale $M$ could be close to the electroweak scale.

In this new scenario, proton stability could be problematic. It is not possible anymore to request that the gauge bosons which mediate baryon number violating (BNV) processes be supermassive [5]. A new mechanism has been suggested in Ref. [6] where leptons and quarks are localized at two different positions in the extra space. The four-dimensional effective coupling of the higher dimension operators responsible for proton decay is then suppressed by the small overlap of the quark and lepton wave functions along the extra dimensions.

It has also been proposed [6] that by localizing different generations, and at the same time placing left and right components at different positions along the extra space, one can obtain in a natural way quark mass matrices which reproduce a realistic mass spectrum and the quark mixing matrix. In Ref. [7] it has been shown that a split fermion scenario, disfavored by constraints on flavor changing neutral currents mediated by Kaluza-Klein gauge bosons, is still possible if the three-dimensional wall has inverse thickness $L^{-1} \geq 100 \mathrm{TeV}$.

Having these scenarios in mind, it is reasonable to ask if BNV processes involving higher generations, or righthanded components, could be faster than the processes involving the first generation, which we know to be strongly suppressed. One can imagine an extra space geography where the second and third generation quarks have a big overlap with the leptons while the first generation quarks are still sufficiently far away from the lepton localization position.

It is therefore important to give an estimate of how fast $\mathrm{BNV}$ processes involving higher generations could be, and how left- or right-handed dynamics may affect them. In Ref. [8] it was suggested that one can estimate a BNV $\tau$ decay branching ratio by using proton decay constraints. Applying the same approach, we estimate BNV processes involving higher generations.

Let us write down all possible dimension six operators that violate the baryon number, but respect the symmetries of the standard model (SM) $[9,10]$ :

$$
\begin{gathered}
O_{a b c d}^{(1)}=\left(\bar{d}_{\alpha a R}^{c} u_{\beta b R}\right)\left(\bar{q}_{i \gamma c L}^{c} \ell_{j d L}\right) \epsilon_{\alpha \beta \gamma} \epsilon_{i j}, \\
O_{a b c d}^{(2)}=\left(\bar{q}_{i \alpha a L}^{c} q_{j \beta b L}\right)\left(\bar{u}_{\gamma c R}^{c} \ell_{d R}\right) \epsilon_{\alpha \beta \gamma} \epsilon_{i j}, \\
O_{a b c d}^{(3)}=\left(\bar{q}_{i \alpha a L}^{c} q_{j \beta b L}\right)\left(\bar{q}_{k \gamma c L}^{c} \ell_{l d L}\right) \epsilon_{\alpha \beta \gamma} \epsilon_{i j} \epsilon_{k l}, \\
O_{a b c d}^{(4)}=\left(\bar{q}_{i \alpha a L}^{c} q_{j \beta b L}\right)\left(\bar{q}_{k \gamma c L}^{c} \ell_{l d L}\right) \epsilon_{\alpha \beta \gamma}(\vec{\tau} \epsilon)_{i j}(\vec{\tau} \epsilon)_{k l} \epsilon_{i k}, \\
O_{a b c d}^{(5)}=\left(\bar{d}_{\alpha a R}^{c} u_{\beta b R}\right)\left(\bar{u}_{\gamma c R}^{c} \ell_{d R}\right) \epsilon_{\alpha \beta \gamma}, \\
O_{a b c d}^{(6)}=\left(\bar{u}_{\alpha a R}^{c} u_{\beta b R}\right)\left(\bar{d}_{\gamma c R}^{c} \ell_{d R}\right) \epsilon_{\alpha \beta \gamma},
\end{gathered}
$$

where $\alpha, \beta, \gamma$ are color indices, $i, j$ are $\mathrm{SU}(2)$ indices, $a, b$, $c, d$ are family indices, and $\epsilon_{\alpha \beta \gamma}, \epsilon_{i j}$ are totally antisymmetric [11]. From Eqs. (1)-(6) one can build the effective interaction 


$$
\sum_{n=1}^{6} C_{a b c d}^{(n)} O_{a b c d}^{(n)}
$$

by introducing the coefficients $C_{a b c d}^{(n)}$ of dimension $d=$ -2 . The coefficients $C_{a b c d}^{(n)}$ are model dependent quantities which are sensitive to high energy scales. We shall give a model independent estimate of the $C_{a b c d}^{(n)}$ coefficients by using experimental limits on nucleon decays.

The structure of this paper is as follows. In Sec. II we estimate BNV $\tau$ decay branching ratios by using proton decay constraints. In Sec. III we apply the same approach to higher generation quarks and consider BNV processes mediated by tree diagrams. In Sec. IV we show that loop diagrams give much stronger constraints. In Sec. V we estimate BNV $D$ and $B$ meson decays. Our conclusions follow.

\section{BNV OPERATORS AND $\tau$ DECAYS}

We start by analyzing BNV processes involving $\tau$, which are intuitively very intriguing. The fact that the proton cannot decay into $\tau$ but only into $\nu_{\tau}$ implies that BNV decays involving left-handed $\tau$ are highly constrained, but for right-handed $\tau$, BNV processes could be in principle faster. However, following Marciano [8], by considering processes with virtual $\tau$, as for example $p \rightarrow$ "virtual $\tau$ " $\rightarrow \bar{\nu}_{\tau} \pi^{+}$(Fig. 1), one can also strongly constrain $\tau$ decays involving right-handed $\tau$. So far the search for $\tau^{-} \rightarrow \bar{p} \pi^{0}, \bar{p} \eta, \bar{p} \pi^{0} \pi^{0}, \bar{p} \pi^{0} \eta, \bar{p} \gamma \quad[12-14]$ and $\Lambda(\bar{\Lambda}) \pi^{-}[14]$ decay modes has given only upper limits.

Let us calculate explicitly the process of Fig. 1 when the BNV vertex involves right-handed $\tau$. We use the chiral Lagrangian obtained from the operators of Eqs. (2), (5), and (6). Each operator transforms under $\mathrm{SU}_{L}(3) \times \mathrm{SU}_{R}(3)$ like $(\overline{3}, 3)$, hence the $\Delta B=1$ Lagrangian is

$$
\alpha_{p}\left(C_{u u d \tau}^{R} \operatorname{TrO} \xi^{\dagger} \overline{\mathbf{B}}_{R}^{c} \xi^{\dagger}+C_{u u s \tau}^{R} \operatorname{TrO} \xi^{\dagger} \overline{\mathbf{B}}_{R}^{c} \xi^{\dagger}\right) \tau_{R},
$$

where $\xi=\exp \left(i M / f_{\pi}\right)$ with $M$ and $\mathbf{B}$ the pseudoGoldstone boson and octet baryon fields, respectively, and $C_{u u d \tau}^{R}, C_{u u s \tau}^{R}$ are effective couplings for operators in Eqs. (2), (5), and (6). The matrices $\mathrm{O}$ and Õ project out

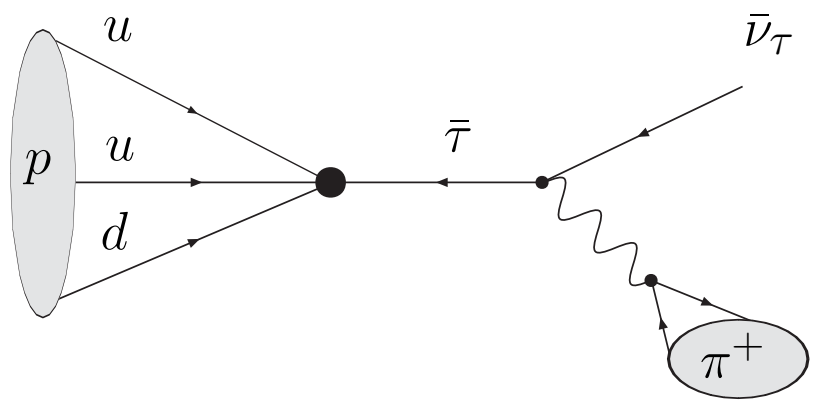

FIG. 1. $\quad p \rightarrow \bar{\nu}_{\tau} \pi^{+}$generated by $C_{u u d \tau}^{R}$.
$\Delta S=0$ and 1 terms $[15,16]$. The strong interaction parameter $\alpha_{p}$ is defined by the relation $\left\langle 0\left|\epsilon_{\alpha \beta \gamma} d_{\alpha R} u_{\beta R} u_{\gamma L}\right| p\right\rangle \equiv \alpha_{p} u_{p L}$, with $u_{p}$ the proton spinor. A recent lattice calculation gives [17] $\alpha_{p}=$ $-0.015 \mathrm{GeV}^{3}$, applicable for proton decay. The BNV vertex in Fig. 1 corresponds to $\alpha_{p} C_{u u d \tau}^{R} \bar{\tau}_{R}^{c} p_{R}$ from Eq. (8), with $\alpha_{p}$ now at the $\tau$ scale. A straightforward calculation for the mode $p \rightarrow \bar{\nu}_{\tau} \pi^{+}$shown in Fig. 1 gives the decay rate

$$
\Gamma=\frac{\alpha_{p}^{2} G_{F}^{2} f_{\pi}^{2}}{4 \pi} \frac{m_{\tau}^{2}\left(m_{p}^{2}-m_{\pi}^{2}\right)^{2}}{m_{p}\left(m_{\tau}^{2}-m_{p}^{2}\right)^{2}}\left|C_{u u d \tau}^{R}\right|^{2},
$$

with $G_{F}$ the Fermi coupling constant. From the experimental limit $\tau\left(p \rightarrow \bar{\nu} \pi^{+}\right)>25 \times 10^{30} \mathrm{yr}$, one can now constrain $C_{u u d \tau}^{R}$ and obtain

$$
C_{u u d \tau}^{R} \lesssim 6.1 \times 10^{-18} \mathrm{TeV}^{-2},
$$

where we have applied the same value for $\alpha_{p}$ as in the proton decay.

Using Eq. (8), the general $\mathrm{SU}_{L}(3) \times \mathrm{SU}_{R}$ (3) invariant chiral Lagrangian involving the $M$ and $\mathbf{B}$ fields [15], and the $\tau$ width [3], we obtain the branching ratios,

$$
\begin{aligned}
& \mathcal{B}\left(\tau \rightarrow \bar{p} \pi^{0}\right) \lesssim 1.6 \times 10^{-4}\left|C_{\text {uиd } \tau}^{R}\right|^{2}, \\
& \mathcal{B}\left(\tau \rightarrow \bar{p} K^{0}\right) \lesssim 7.5 \times 10^{-5}\left|C_{\text {uus }}^{R}\right|^{2}, \\
& \mathcal{B}\left(\tau \rightarrow \bar{\Lambda} \pi^{-}\right) \lesssim 1.3 \times 10^{-4}\left|C_{\text {uus } \tau}^{R}\right|^{2},
\end{aligned}
$$

where $C_{u u d \tau}^{R}, C_{u u s \tau}^{R}$ are in $\mathrm{TeV}^{-2}$ units. Substituting Eq. (10) in Eq. (11) one obtains

$$
\mathcal{B}\left(\tau \rightarrow \bar{p} \pi^{0}\right) \lesssim 5.9 \times 10^{-39} .
$$

The other two modes involving a strange quark depend on the effective coupling $C_{u u s \tau}^{R}$, which corresponds to the $-i \alpha_{p} / f_{\pi}\left(C_{u u s \tau}^{R} \bar{\tau}_{R}^{c} p_{R} K^{0 \dagger}\right)$ term from Eq. (8), with $\alpha_{p}$ again at the $\tau$ scale. Considering the process $p \rightarrow$ $K^{0} e^{+} \nu_{e} \bar{\nu}_{\tau}$ of Fig. 2, and using $\tau>0.6 \times 10^{30} \mathrm{yr}$ for the semi-inclusive process $p \rightarrow e^{+}+$anything, one obtains

$$
C_{\text {uus } \tau}^{R} \lesssim 1.8 \times 10^{-13} \mathrm{TeV}^{-2},
$$

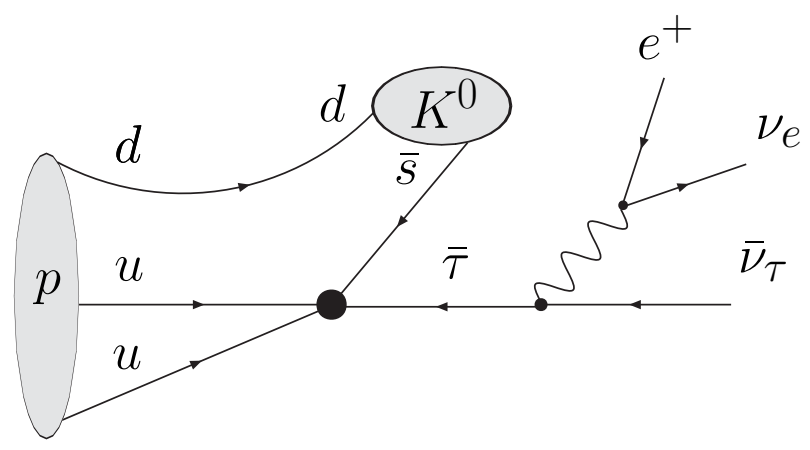

FIG. 2. $\quad p \rightarrow K^{0} e^{+} \nu_{e} \bar{\nu}_{\tau}$ generated by $C_{u u s \tau}^{R}$. 
where again we have applied the same value for $\alpha_{p}$ as in the proton decay. Substituting now Eq. (15) in Eqs. (12) and (13) we also obtain

$$
\begin{gathered}
\mathcal{B}\left(\tau \rightarrow \bar{p} K^{0}\right) \lesssim 1.4 \times 10^{-30}, \\
\mathcal{B}\left(\tau \rightarrow \bar{\Lambda} \pi^{-}\right) \lesssim 2.3 \times 10^{-30} .
\end{gathered}
$$

One can generate $\tau \rightarrow \bar{p} \gamma, \bar{\Sigma}^{+} \gamma$ decays from Eq. (8) by radiating a photon off the external lines in the effective $\tau \rightarrow \bar{p}, \bar{\Sigma}^{+}$transitions, which are analogous to $p \rightarrow \ell^{+} \gamma$. Direct radiation from the transition vertex would involve higher dimension operators, hence suppressed. The radiative process should be down by a power of $\alpha_{\mathrm{em}} / \pi$ compared to the modes we have just discussed. Indeed, we find the branching fraction,

$$
\mathcal{B}(\tau \rightarrow \bar{p} \gamma) \lesssim 2.8 \times 10^{-7}\left|C_{\text {uиd } \tau}^{R}\right|^{2} \lesssim 1.0 \times 10^{-41} .
$$

We see that BNV $\tau$ decays are extremely suppressed, and it is unlikely that we will ever observe them at any future machine. We now turn to BNV processes involving higher generation quarks.

\section{III. $C_{t t(c) b \ell}$ COUPLING FROM $\boldsymbol{n} \rightarrow \ell^{+} \boldsymbol{\pi}^{+} \boldsymbol{\pi}^{-} \boldsymbol{\pi}^{-}$}

In the previous section we showed how Marciano's suggestion of considering virtual $\tau$ in proton decays can be used to constrain $C_{u u d \tau}^{R}$ and $C_{u u s \tau}^{R}$. By analogy one can then constrain any coupling $C_{a b c d}$ through nucleon decays, where now the family indices $a, b, c$, and $d$ can belong to any generation.

As an example, we give in Fig. 3 the tree diagram for $n \rightarrow \ell^{+}\left(p_{1}\right) \pi^{+}\left(p_{2}\right) \pi^{-}\left(p_{3}\right) \pi^{-}\left(p_{4}\right)$ induced by a $C_{t t b \ell}$ coupling, where $\ell^{+}$could be $e^{+}$or $\mu^{+}$. As internal states all possible combinations are allowed, where a $b(t)$ quark propagator can be replaced by a $d$ or $s(u$ or $c$ ) quark. The amplitude will then be a combination of terms each proportional to a different $C_{a b c d}$.

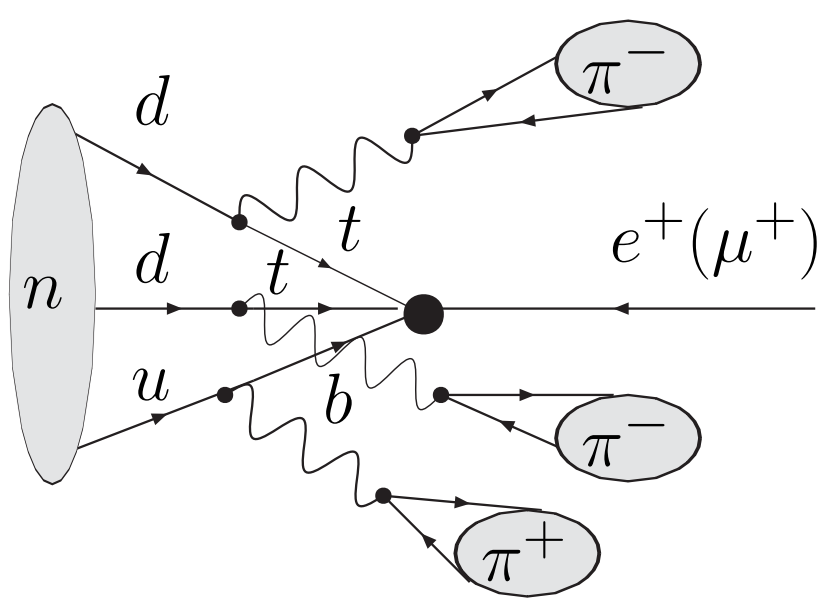

FIG. 3. $n \rightarrow \pi^{+} \pi^{-} \pi^{-} e^{+}\left(\mu^{+}\right)$generated by $C_{t t b \ell}$.
As we are interested in giving only an order of magnitude estimate, we can use $\tau\left(n \rightarrow e^{+}\left(\mu^{+}\right)+\right.$anything $)>$ $0.6(12) \times 10^{30} \mathrm{yr}$ to constrain the $C_{a b c d}$ 's by assuming each time that only one single term dominates. Taking, for example, the term proportional to $C_{t t b \ell}$ as the dominant contribution and considering for simplicity only the effect of $O_{t t b \ell}^{(5)}$, the amplitude can be written as

$$
\begin{aligned}
\mathcal{M}_{n \rightarrow \ell \pi \pi \pi}^{(5)}= & \frac{G_{F}^{3} f_{\pi}^{3}}{2 \sqrt{2}} V_{u b}^{*} V_{t d}^{2} C_{t t b \ell}^{(5)} \\
& \times\left\langle\ell^{+} \pi^{-} \pi^{+} \pi^{-}\right|\left[\bar{u}^{c} \not p_{2}\left(1+\gamma_{5}\right) b^{c}\right]\left[\bar{b}_{R}^{c} t_{R} \bar{t}_{R}^{c} \ell_{R}\right] \\
& \times\left[\bar{d}^{c} \not p_{3}\left(1+\gamma_{5}\right) t^{c}\right]\left[\bar{t} \not p_{4}\left(1-\gamma_{5}\right) d\right]|n\rangle
\end{aligned}
$$

Using the matrix element $\left\langle 0\left|\bar{u}^{c} \bar{d}^{c} d\right| n\right\rangle \equiv \alpha_{n} \bar{v}_{n}$, we obtain

$$
\begin{aligned}
i \mathcal{M}_{n \rightarrow \ell \pi \pi \pi}^{(5)}= & 2 \sqrt{2} G_{F}^{3} f_{\pi}^{3} V_{u b}^{*} V_{t d}^{2} \alpha_{n} C_{t t b \ell}^{(5)} \frac{1}{m_{b} m_{t}^{2}} \\
& \times \operatorname{tr}\left(\not \not_{2} \not \not_{4}\right) \bar{v}_{n} \not \not_{3}\left(1+\gamma_{5}\right) v_{\ell},
\end{aligned}
$$

where we have neglected the internal quark momenta $p_{b}$, $p_{t}$, and $p_{t}^{\prime}$ [all of $O(1 \mathrm{GeV})$ ] with respect to $m_{b}(1 \mathrm{GeV})$ and $m_{t}(1 \mathrm{GeV})$.

It is clear from Eq. (20) that the process $n \rightarrow$ $\ell^{+} \pi^{+} \pi^{-} \pi^{-}$is highly suppressed, depending on the 6th power of $G_{F}$ and the 18th power of the Cabibbo parameter $l$ appearing in the Wolfenstein parametrization of the Cabibbo-Kobayashi-Maskawa matrix. Further suppression comes from four body phase space and the hadronic parameter $\alpha_{n}$. Thus, the experimental constraint $\tau(n \rightarrow$ $e^{+}\left(\mu^{+}\right)+$anything $)>0.6(12) \times 10^{30} \mathrm{yr}$ can be satisfied without requiring the effective coupling $C_{t t b \ell}^{(5)}$ to be very small. In fact $C_{t t b \ell}^{(5)}$ can be huge. We obtain for the $n \rightarrow$ $\ell^{+} \pi^{+} \pi^{-} \pi^{-}$rate the following estimate:

$$
\Gamma_{n \rightarrow \ell \pi \pi \pi}^{(5)}[\mathrm{TeV}] \sim 4 \times 10^{-87}\left|C_{t t b \ell}^{(5)}\right|^{2},
$$

which gives

$$
C_{t t b \ell}^{(5)} \lesssim 10^{11} \mathrm{TeV}^{-2} .
$$

Obviously, the upper limit in Eq. (22) is much higher than what could be realistic, but it would still seemingly suggest that BNV processes like $t \rightarrow \bar{b} \bar{b} W^{-} \ell^{+}$could take place rather quickly. Assuming now that $O_{t c b \ell}^{(i)}$ can give the dominant contribution, from Eq. (20) it is clear that the constraint on $C_{t c b \ell}$ is also very loose, and we obtain the rough estimate

$$
C_{t c b \ell} \sim C_{t t b \ell} \frac{V_{t d}}{V_{c d}} \frac{m_{c}}{m_{t}} \lesssim 10^{7} \mathrm{TeV}^{-2} .
$$

Decays like $t \rightarrow \bar{c} \bar{b} \ell^{+}$could then easily compete with SM top decays making CERN LHC a BNV machine.

Before getting carried away, we remind ourselves that the above discussion of Fig. 3 is just an example. What we need to do is to identify the fastest possible process generated by BNV involving higher generation quarks. It is 
clear that we need to reduce the powers of $G_{F}$ or $\lambda$. We now turn to what we feel is the best process in the next section.

\section{IV. $C_{t t(c) b \ell}$ COUPLING FROM $\boldsymbol{n} \rightarrow \bar{\nu} \pi^{0}$}

The process $n \rightarrow \ell^{+} \pi^{+} \pi^{-} \pi^{-}$in Fig. 3 is highly suppressed, because for each tree level virtual $W$ emission, there is one power of $G_{F}$ suppression. It is clear that a diagram involving $W$ emission at loop level will be less suppressed. In Fig. 4 we give the two-loop radiative corrections for the process $n \rightarrow \bar{\nu}_{\tau} \pi^{0}$ involving $C_{t t b \tau}$; similar diagrams describe two-loop radiative corrections for the process $n \rightarrow \bar{\nu}_{e, \mu} \pi^{0}$ with $\tau$ replaced by $e$ and $\mu$. The amplitude for the process $n \rightarrow \bar{\nu}_{\tau} \pi^{0}$ at tree level is proportional to $C_{u d d \nu_{\tau}}$. When radiative corrections are considered, $C_{u d d \nu_{\tau}}$ mixes with all the other couplings including $C_{t t b \tau}$. Knowing that $C_{u d d \nu_{\tau}}$ is already constrained from the tree level calculation, we obtain a rough estimate of $C_{t t b \tau}$ by just isolating the contribution to the amplitude given by the diagrams of Fig. 4. We find in this way the most stringent constraint on $C_{t t(c) b \ell}^{(i)}$. A very similar approach has been used in Ref. [18] to constrain the gauge couplings of an $\mathrm{SU}(2)_{1} \times \mathrm{SU}(2)_{2}$ extension of the standard model. This theory can in fact give rise to baryon and lepton number violating processes via the instanton transition associated with the extended gauge group.

Again we are interested only in order of magnitude results, and it is therefore unnecessary to sum up all amplitudes coming from different operators. Let us assume, for example, that $C_{t t b \ell}^{(3)}$ gives the dominant contribution by considering only the effect of the operator $O_{t t b \ell}^{(3)}$ involving left-handed components. The amplitude for the first diagram in Fig. 4 gives

$$
\begin{aligned}
\mathcal{M}_{n \rightarrow \bar{\nu} \pi^{0}}^{(3)}= & \frac{g^{4}}{64} V_{u b}^{*} V_{t d}^{2} C_{t t b \ell}^{(3)} \int \frac{d^{4} k_{1} d^{4} k_{2}}{(2 \pi)^{4}(2 \pi)^{4}} \\
& \times \frac{-g_{\mu \nu} g_{\alpha \beta}}{\left(k_{1}^{2}-M_{W}^{2}\right)\left(k_{2}^{2}-M_{W}^{2}\right)}\left\langle\bar{\nu} \pi^{0}\right|\left[\bar{t} \gamma^{\mu}\left(1-\gamma^{5}\right) d\right] \\
& \times\left[\bar{d}^{c} \gamma^{\alpha}\left(1+\gamma^{5}\right) t^{c}\right]\left[\bar{u}^{c} \gamma^{\beta}\left(1+\gamma^{5}\right) b^{c}\right] \\
& \times\left[\bar{e} \gamma^{\nu}\left(1-\gamma^{5}\right) \nu\right]\left[\bar{b}_{L}^{c} t_{L} \bar{t}_{L}^{c} \ell_{L}\right]|n\rangle .
\end{aligned}
$$

Using the matrix element $\left\langle\pi^{0}\left|\bar{u}^{c} \bar{d}^{c} d\right| n\right\rangle \equiv \alpha_{n \pi} \bar{v}_{n}$ [19], and after some simplifications, we get

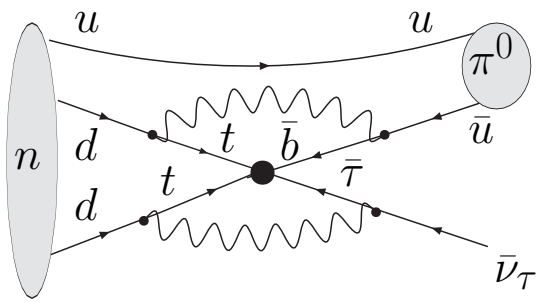

$$
\begin{aligned}
\mathcal{M}_{n \rightarrow \bar{\nu} \pi^{0}}= & -2 G_{F}^{2} M_{W}^{4} V_{u b}^{*} V_{t d}^{2} \alpha_{n \pi} C_{t t b \ell}^{(3)} \int \frac{d^{4} k_{1} d^{4} k_{2}}{(2 \pi)^{4}(2 \pi)^{4}} \\
& \times \frac{\operatorname{tr}\left[\gamma^{\alpha} \not p_{b} p_{t} \gamma^{\mu}\left(1-\gamma^{5}\right)\right]}{\left(k_{1}^{2}-M_{W}^{2}\right)\left(k_{2}^{2}-M_{W}^{2}\right)} \\
& \times \frac{\bar{v}_{n} \gamma_{\alpha} \not_{t}^{\prime} p_{l} \gamma_{\mu}\left(1-\gamma_{5}\right) v_{\nu}}{\left(p_{b}^{2}-m_{b}^{2}\right)\left(p_{t}^{2}-m_{t}^{2}\right)\left(p_{t}^{\prime 2}-m_{t}^{2}\right)\left(p_{\ell}^{2}-m_{\ell}^{2}\right)},
\end{aligned}
$$

where $k_{1}$ and $k_{2}$ are the momenta of the internal $W$ bosons, and $p_{b}, p_{t}, p_{t}^{\prime}$, and $p_{\ell}$ are the momenta of internal quarks and leptons.

Note that our BNV interactions are of four fermion interaction form, hence not renormalizable. The two loops integral in Eq. (25) is ultraviolet divergent. To obtain a finite result we can introduce a cutoff scale $\Lambda$ where we assume our effective theory will break down. Setting all the external momenta to zero we obtain

$$
\begin{aligned}
\mathcal{M}_{n \rightarrow \bar{\nu} \pi^{0}}= & -2 G_{F}^{2} M_{W}^{4} V_{u b}^{*} V_{t d}^{2} \alpha_{n \pi} C_{t t b b}^{(3)} I^{(1) \varrho \sigma} I^{(2) \tau \omega} \\
& \times \operatorname{tr}\left[\gamma^{\alpha} \gamma_{\tau} \gamma_{\varrho} \gamma^{\mu}\left(1-\gamma^{5}\right)\right] \\
& \times \bar{v}_{n} \gamma_{\alpha} \gamma_{\omega} \gamma_{\sigma} \gamma_{\mu}\left(1-\gamma_{5}\right) v_{\nu}
\end{aligned}
$$

where $I^{(i) \alpha \beta}$ is defined as

$$
I^{(i) \alpha \beta} \equiv \int \frac{d^{4} k_{i}}{(2 \pi)^{4}} \frac{k_{i}^{\alpha} k_{i}^{\beta}}{\left(k_{i}^{2}-m_{a}^{2}\right)\left(k_{i}^{2}-m_{b}^{2}\right)\left(k_{i}^{2}-M_{W}^{2}\right)},
$$

with $\left(m_{a}, m_{b}\right)$, respectively, $\left(m_{t}, m_{\ell}\right)$ and $\left(m_{b}, m_{t}\right)$. The integral $I^{(i) \alpha \beta}$ has logarithmic dependence in the cutoff scale $\Lambda$ plus corrections of order $\Lambda^{-2}$ [18]. Setting the scale $\Lambda=10 \mathrm{TeV}$, and considering only the contribution to the amplitude coming from $O_{t t b \ell}^{(3)}$, we obtain the following rough estimate for the $n \rightarrow \bar{\nu} \pi^{0}$ rate:

$$
\Gamma_{n \rightarrow \bar{\nu} \pi^{0}}^{(3)}[\mathrm{TeV}] \lesssim 10^{-38}\left|C_{t t b \ell}^{(3)}\right|^{2},
$$

where $C_{t t b \ell}^{(3)}$ is measured in $\mathrm{TeV}^{-2}$. Using the experimental lower limit on the $n \rightarrow \bar{\nu} \pi^{0}$ partial lifetime, $\tau_{n \rightarrow \bar{\nu} \pi^{0}}>$ $112 \times 10^{30} \mathrm{yr}$, we obtain

$$
C_{t t b \ell}^{(3)} \lesssim 10^{-14} \mathrm{TeV}^{-2} .
$$

One can follow the same approach for the operator $O_{t t b \ell}^{(5)}$ which involves only right-handed fields [20], allowing for a

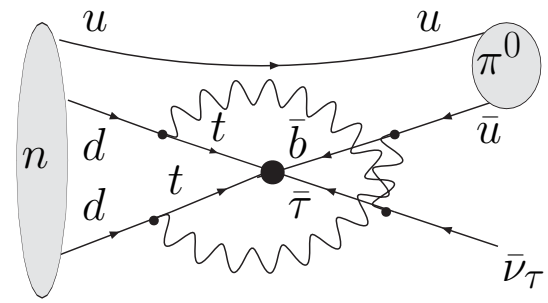

FIG. 4. $n \rightarrow \bar{\nu}_{\tau} \pi^{0}$ generated by $C_{t t b \tau}$. 
weaker constraint on $C_{t t b \ell}^{(5)}$

$$
C_{t t b \ell}^{(5)} \lesssim 10^{-11} \mathrm{TeV}^{-2} \text {. }
$$

Assuming now that $O_{\text {tcbe }}^{(i)}$ gives the dominant contribution and using Eqs. (29) and (30) we obtain

$$
C_{t c b \ell}^{(3)} \sim C_{t t b \ell}^{(3)} \frac{V_{t d}}{V_{c d}} \frac{I\left(m_{b}, m_{t}\right)}{I\left(m_{b}, m_{c}\right)} \lesssim 10^{-16} \mathrm{TeV}^{-2},
$$

with $I\left(m_{a}, m_{b}\right)$ defined by $I^{\alpha \beta}\left(m_{a}, m_{b}\right) \equiv g^{\alpha \beta} I\left(m_{a}, m_{b}\right)$, and

$$
C_{t c b \ell}^{(5)} \sim C_{t t b \ell}^{(5)} \frac{V_{t d}}{V_{c d}} \frac{m_{t}}{m_{c}} \frac{I_{S}\left(m_{b}, m_{t}\right)}{I_{S}\left(m_{b}, m_{c}\right)} \lesssim 10^{-11} \mathrm{TeV}^{-2},
$$

where

$$
I_{S} \equiv \int \frac{d^{4} k}{(2 \pi)^{4}} \frac{1}{\left(k^{2}-m_{a}^{2}\right)\left(k^{2}-m_{b}^{2}\right)\left(k^{2}-M_{W}^{2}\right)} .
$$

From Eq. (32) we expect $t \rightarrow \bar{c} \bar{b} \ell^{+}$can have at most a branching ratio

$$
\mathcal{B}\left(t \rightarrow \bar{c} \bar{b} \ell^{+}\right) \sim 10^{-5}\left|C_{t c b \ell}^{(5)}\right|^{2} \lesssim 10^{-27},
$$

where $C_{t t b \ell}^{(5)}$ is in $\mathrm{TeV}^{-2}$. This excludes any possibility for discovering BNV top decays at future colliders.

\section{V. $D$ AND $B$ DECAYS}

In this section we will give rough estimates for BNV $D$ and $B$ meson decay branching ratios. Following the arguments of the previous sections for BNV $\tau$ and top quark decays we expect BNV $D$ and $B$ processes to be also very suppressed. Nevertheless we will still try to give some directions for a possible search.

We start by considering $D$ decays. We first note that, to allow for a baryon in the final state, the $\tau$ lepton is forbidden, hence we consider only $c \rightarrow \ell^{+} \bar{s} \bar{u}, \ell^{+} \bar{d} \bar{u}$ processes with $\ell=e, \mu$. These lead to the decays $D^{+} \rightarrow \bar{\Lambda} \ell^{+}\left(\bar{\Delta}^{0} \ell^{+}\right.$ has more background) and $D^{0} \rightarrow \bar{\Sigma}^{+} \ell^{+}, \bar{p} \ell^{+}$. The $D^{+}$ mode has some advantage because it has a longer lifetime and cleaner signature. Since we are not concerned with hadronic effects, we emulate the Lagrangian for $\tau$ decay, Eq. (8), which is itself inspired by the chiral Lagrangian for proton decay. Assuming that hadronic factors are of the same order of magnitude, we find the Lagrangian for $D$ meson decays of interest,

$$
\begin{aligned}
& i \frac{\alpha_{D}}{f_{D}} C_{u c d \ell}^{R} D^{0}\left(\bar{p}_{R}^{c} \ell_{R}\right)+i \frac{\alpha_{D}}{f_{D}} C_{u c s \ell}^{R}\left(D^{0}\left(\bar{\Sigma}_{R}^{+c} \ell_{R}\right)\right. \\
& \left.+\sqrt{\frac{2}{3}} D^{+}\left(\bar{\Lambda}_{R}^{c} \ell_{R}\right)\right) .
\end{aligned}
$$

From this we obtain the branching ratios,

$$
\mathcal{B}\left(D^{+} \rightarrow \bar{\Lambda} \ell^{+}\right) \lesssim 3.9 \times 10^{-4}\left|C_{u c s \ell}^{R}\right|^{2},
$$

$$
\begin{gathered}
\mathcal{B}\left(D^{0} \rightarrow \bar{\Sigma}^{+} \ell^{+}\right) \lesssim 3.1 \times 10^{-4}\left|C_{u c s \ell}^{R}\right|^{2}, \\
\mathcal{B}\left(D^{0} \rightarrow \bar{p}^{+}\right) \lesssim 1.1 \times 10^{-4}\left|C_{u c d \ell}^{R}\right|^{2},
\end{gathered}
$$

where the $C$ 's are in units of $\mathrm{TeV}^{-2}$, and we have set $\alpha_{D} \lesssim$ $\alpha_{p}$. These single baryon plus lepton final states are rather distinct, but have so far never been searched for. In fact, baryon number conservation instructs one to consider a baryon pair in the final state, which is kinematically forbidden. Thus, baryons in $D$ decay final states have commonly been ignored. $D$ decay to final states containing a baryon, however, only probes baryon number violation in second and first generations.

We do not consider $D_{s}$ decays, as it offers no special advantage as a probe. We mention, however, the $\Lambda_{c}$ baryon, where the process $\Lambda_{c} \rightarrow \tau^{+} K^{0}$ allows for $\tau$ in the final state. The process in principle probes $c u s \rightarrow \tau^{+}$ interaction. However, experimental study poses a problem. Not only would $\Lambda_{c}$ be hadronically produced, having a $\tau$ in the final state does not allow for full reconstruction. For this reason, we shall drop $\tau$ from consideration even if it is kinematically allowed in the decay final state. For a rough estimate of the branching ratios in Eqs. (36)-(38), we use for $C_{u c s \ell}^{R}$ and $C_{u c d \ell}^{R}$, respectively, $C_{u u s \tau}^{R}$ and $C_{u u d \tau}^{R}$ from Eqs. (10) and (15) obtaining

$$
\begin{aligned}
& \mathcal{B}\left(D^{+} \rightarrow \bar{\Lambda} \ell^{+}\right) \lesssim 1.3 \times 10^{-29}, \\
& \mathcal{B}\left(D^{0} \rightarrow \bar{\Sigma}^{+} \ell^{+}\right) \lesssim 1.0 \times 10^{-29}, \\
& \mathcal{B}\left(D^{0} \rightarrow \bar{p} \ell^{+}\right) \lesssim 4.0 \times 10^{-39} .
\end{aligned}
$$

We now turn to the $B$ system. Having dropped $\tau$ from the final state, the transitions of interest are $\bar{b} \rightarrow c c \ell, c u \ell$, $u u \ell$, where $\ell=\mu, e$. In a way, $\bar{b} \rightarrow \mu c c$ is analogous to $b \rightarrow \bar{s} s s$, as far as generation counting is concerned. This provides some extra motivation, as $C P$ violation in $B \rightarrow$ $\phi K_{S}$ could be hinting at the presence of new physics. One can in principle have the double charmed baryon $\Xi_{c c}$ in the final state, i.e. $B^{0,+} \rightarrow \Xi_{c c}^{+,++} \ell^{-}$. The $\Xi_{c c}^{+}$state is seen [21] in the $\Lambda_{c}^{+} K^{-} \pi^{+}$channel, although it still needs to be confirmed. By analogy with $D^{+} \rightarrow K^{-} \pi^{+} \pi^{+}$which has $\sim 9 \%$ rate, we expect $\mathcal{B}\left(\Xi_{c c}^{+} \rightarrow \Lambda_{c}^{+} K^{-} \pi^{+}\right)$could be of order $20 \%$. Since the $c c$ system is generated from the $\bar{b} \rightarrow$ $c c \ell$ transition with nonrelativistic motion, there should be little suppression in the $B \rightarrow \Xi_{c c}$ form factor. Again, we make an analogy with $\tau \rightarrow \bar{\Lambda} \pi^{-}, D \rightarrow \bar{\Lambda} \ell^{+}$couplings of Eqs. (8) and (35), and estimate the branching ratio as

$$
\mathcal{B}\left(B^{0,+} \rightarrow \Xi_{c c}^{+,++} \ell^{-}\right) \lesssim 1.0 \times 10^{-4}\left|C_{c c b \ell}^{R}\right|^{2} .
$$

The equivalent $\alpha_{B \rightarrow \Xi_{c c}}$ factor is set to $\alpha_{p}$ value. Using Eq. (32) we obtain 


$$
C_{c c b \mu}^{(5)} \sim C_{t c b \tau}^{(5)} \frac{V_{t d}}{V_{c d}} \frac{m_{t}}{m_{c}} \frac{I_{S}\left(m_{t}, m_{\tau}\right)}{I_{S}\left(m_{c}, m_{\mu}\right)} \lesssim 10^{-12} \mathrm{TeV}^{-2},
$$

and from Eqs. (42) and (43) we can estimate $\mathcal{B}\left(B^{0,+} \rightarrow\right.$ $\left.\Xi_{c c}^{+,+} \ell^{-}\right) \lesssim 1.0 \times 10^{-28}$.

Although $\Xi_{c c}$ decay is likely dominated by only a few channels, its reconstruction is still a problem. Typical $\Lambda_{c}$ decay modes are a few percent, so one would be limited by $\Lambda_{c}$ reconstruction efficiency. Perhaps one can also consider double charm $(\Delta C=2)$ final states such as $B^{+} \rightarrow$ $D^{*+} D^{0} p \ell^{-}$, which is quite close to threshold, but would be stunning if seen: proton, $\ell^{-}$and (slow) $\pi^{+}$from $B$ vertex, while one has two $D$, rather than $D \bar{D}$, tags. One could also consider the modes $\bar{B} \rightarrow \Lambda_{c}^{+} D^{(*) 0,+} \ell^{-}$. Again, it would be hard to set a bound on the effective coupling, but it can be searched for.

For $\bar{b} \rightarrow c u \ell$ generated processes such as $B^{0} \rightarrow \Lambda_{c}^{+} \ell^{-}$, estimation becomes unreliable because of the relatively large energy release [22], and the $\Lambda_{c}$ would be in relativistic motion in the $B$ decay frame. But if we continue with our approach, the branching ratio is

$$
\mathcal{B}\left(B^{0} \rightarrow \Lambda_{c}^{+} \ell^{-}\right)<1.1 \times 10^{-4}\left|C_{u c b \ell}^{R}\right|^{2},
$$

where $\alpha_{B \rightarrow \Lambda_{c}}<\alpha_{p}$ is applied. The suppression from $\alpha_{B \rightarrow \Lambda_{c}}^{2} / \alpha_{p}^{2}$ could be as large as 100 , if one compares the observed $\bar{B}^{0} \rightarrow \Lambda_{c} \bar{p}$ rate [23] with that of $D^{+} \pi^{-}$. This would give $\mathcal{B}\left(B^{0} \rightarrow \Lambda_{c}^{+} \ell^{-}\right) \lesssim 4 \times 10^{-30}$, if one applies the bound of Eq. (15).

What may be more reasonable for the $B$ system, in view of the large available energy, is to adopt an inclusive approach. Note that $\bar{b} \rightarrow c u \ell^{-}$bears some similarity with the usual $\bar{b} \rightarrow \bar{c} \ell^{+} \nu$, except for the wrong charge combination for $c \ell^{-}$vs $\bar{c} \ell^{+}$arising from $\bar{b}$ decay (plus replacing the $\nu$ by the $u$ quark). With $b$ tagging, one searches for $B \rightarrow \Lambda_{c}^{+} \ell^{-} X$, with no missing energy. If this can be properly achieved, it can be equated with the inclusive $\bar{b} \rightarrow c u \ell^{-}$branching ratio,

$$
\mathcal{B}\left(\bar{b} \rightarrow c u \ell^{-}\right)=4.0 \times 10^{-2}\left|C_{u c b \ell}^{R}\right|^{2},
$$

and derive a more stringent bound. Applying the bound of Eq. (15), we find $\mathcal{B}\left(\bar{b} \rightarrow c u \ell^{-}\right) \lesssim 1.3 \times 10^{-27}$. We remark that for $\bar{b} \rightarrow c u \ell^{-}$, because of unequal flavor index, the $O^{3}$ operator of Eq. (3) contributes.
For inclusive $\bar{b} \rightarrow u u \ell^{-}$transitions, we get

$$
\mathcal{B}\left(\bar{b} \rightarrow u u \ell^{-}\right)=7.4 \times 10^{-2}\left|C_{u u b \ell}^{R}\right|^{2}
$$

Exclusive search for $B \rightarrow p \ell^{-}$can be done, but would not be profitable as this mode would be highly form factor suppressed. This is because of the large energy release [22] and highly relativistic proton motion. However, what one should search for is $B \rightarrow p \ell^{-} X$ with $b$ tagging, where $p \ell^{-}$ has the wrong charge combination and come from the $B$ decay vertex. Charm veto for semileptonic decays should be applied. The study would probably be background limited due to mistag and vertex resolutions, but certainly should be pursued. The limit from Eq. (46), after applying the bound of Eq. (15), is $2.4 \times 10^{-27}$.

\section{CONCLUSION}

We have used experimental data on nucleon decays to give an estimate of BNV couplings involving higher generation leptons and quarks. Using an approach very similar to the one proposed by Marciano to infer a very small branching ratio for BNV $\tau$ decays, we find that BNV processes involving higher generation quarks are also extremely suppressed.

Despite our findings, we believe it is still worthy to look for BNV processes in $\tau$, charm, $B$, and maybe in the future in top decays. Although we find that all BNV processes involving $\tau$ and higher generation quarks are too strongly suppressed by proton stability, such that there seems to be no hope for observation at any future machine, redundancy is very important. In this vein, we note that the CLEO Collaboration performed $\tau^{-} \rightarrow \bar{p} \pi^{0}, \bar{p} \eta, \bar{p} \pi^{0} \pi^{0}, \bar{p} \pi^{0} \eta$, $\bar{p} \gamma$ [13] search after the remark made by Marciano. The search has been extended recently to $\tau^{-} \rightarrow \Lambda(\bar{\Lambda}) \pi^{-}$[14] by the Belle Collaboration.

\section{ACKNOWLEDGMENTS}

This work is supported in part by NSC-94-2112-M-002035, NSC94-2811-M-002-053, and HPRN-CT-200200292. W. S. H. thanks the SLAC Theory Group for hospitality. A. S. would like to thank Hsiang-nan Li and Diego Guadagnoli for very useful discussions.
[1] H. Georgi and S. L. Glashow, Phys. Rev. Lett. 32, 438 (1974).

[2] A. D. Sakharov, Pis'ma Zh. Eksp. Teor. Fiz. 5, 32 (1967) [JETP Lett. 5, 24 (1967)].

[3] S. Eidelman et al. (Particle Data Group), Phys. Lett. B 592, 1 (2004).
[4] N. Arkani-Hamed, S. Dimopoulos, and G. Dvali, Phys. Lett. B 429, 263 (1998).

[5] I. Dorsner and P. F. Pérez, Phys. Lett. B 625, 88 (2005), for model independent lower limits on the gauge bosons mass in the context of grand unified theories with the standard model matter content. 
[6] N. Arkani-Hamed and M. Schmaltz, Phys. Rev. D 61, 033005 (2000).

[7] A. Delgado, A. Pomarol, and M. Quiros, J. High Energy Phys. 01 (2000) 030.

[8] W. J. Marciano, Nucl. Phys. B, Proc. Suppl. 40, 3 (1995).

[9] S. Weinberg, Phys. Rev. Lett. 43, 1566 (1979).

[10] F. Wilczek and A. Zee, Phys. Rev. Lett. 43, 1571 (1979).

[11] All interactions in Eqs. (1)-(6) conserve the difference of baryon and lepton number $B-L$, which implies that nucleons can decay only to antileptons but not leptons.

[12] H. Albrecht et al. (ARGUS Collaboration), Z. Phys. C 55, 539 (1992).

[13] R. Godang et al. (CLEO Collaboration), Phys. Rev. D 59, 091303 (1999).

[14] Y. Miyazaki et al. (Belle Collaboration), hep-ex/0508044 [Phys. Lett. B (to be published)].
[15] M. Claudson, M. B. Wise, and L. J. Hall, Nucl. Phys. B195, 297 (1982).

[16] S. Chada and M. Daniel, Nucl. Phys. B229, 105 (1983).

[17] S. Aoki et al., Phys. Rev. D 62, 014506 (2000).

[18] D. E. Morrisey, T. M. P. Tait, and C. E. M. Wagner, hep-ph/ 0508123 [Phys. Rev. D (to be published)].

[19] We assume the form factor $\alpha_{n \pi} \lesssim \alpha_{n} / f_{\pi}$.

[20] Because of the different chiral structure of the BNV operator $O^{(5)}$ with respect to $O^{(7)}$, the amplitude is finite and no cutoff is required.

[21] M. Mattson et al. (SELEX Collaboration), Phys. Rev. Lett. 89, 112001 (2002).

[22] W. S. Hou and A. Soni, Phys. Rev. Lett. 86, 4247 (2001).

[23] K. Abe et al. (Belle Collaboration), Phys. Rev. Lett. 90, 121802 (2003). 